\title{
Lorentz Invariance Violation and the QED formation length
}

\author{
H. Vankov and T. Stanev \\ Institute for Nuclear Research and Nuclear Energy, Sofia, Bulgaria \\ Bartol Research Institute, University of Delaware, Newark DE 19716, USA
}

\begin{abstract}
It was recently suggested that possible small volations of Lorentz invariance could explain the existence of UHECR beyond the GZK cutoff and the observations of multi-TeV gamma-rays from Mkn 501. Our analysis of Lorentz-violating kinematics shows that in addition to the modified threshold conditions solving cosmic ray puzzles we should expect a strong suppression of electromagnetic processes like bremsstrahlung and pair creation. This leads to drastic effects in electron-photon cascade development in the atmosphere and in detectors.
\end{abstract}

\section{Introduction}

A tiny Lorentz invariance violation (LIV) at high energies was suggested [1] to explain the most discussed at present experimental astrophysical paradoxes the observations of ultra high energy cosmic rays (UHECR) well beyond the theoretically expected Greisen-Zatzepin-Kuzmin (GZK) cutoff around 6x10 19 $\mathrm{eV}[2]$ and the observations of $20 \mathrm{TeV}$ gamma-rays from Mkn 501. UHECR should have been absorbed in photoproduction collisions with the microwave background and the $20 \mathrm{TeV} \gamma$-rays - on the extragalactic infrared/optical background [3]. While some authors [4,5] consider the second puzzle not so dramatic and LIV hypothesis too premature, the number of showers above $10^{20} \mathrm{eV}$ is already big enough to suggest existing of a problem. As a solution of this problem LIV was first suggested about 30 years ago [6,7] and later in [8]. In [1], [9], [10], [11] LIV hypothesis was suggested as a solution for both paradoxes.

A small possible violation of LI was proposed in [9] which corresponds to an 
energy dependent photon group velocity

$$
\frac{\partial E}{\partial k}=c\left[1-\xi_{\gamma} \frac{E}{E_{0}}\right]
$$

Here $c$ is the speed of light. This corresponds to a dispersion relation

$$
c^{2} k^{2} \simeq E^{2}+\xi_{\gamma} \frac{E^{3}}{E_{0}}
$$

with $\xi_{\gamma}= \pm 1$ and $E_{0} \sim 10^{19} \mathrm{GeV}$.

Kifune[10] has used this possibility to investigate the consequences of LIV on collisions of high energy radiation with soft photons. The detection of $\mathrm{TeV}$ photons from point sources and protons above the GZK cutoff sets some constraints on $\xi_{\gamma}, \xi_{e}$ and $\xi_{p}$, when photons, electrons and protons are allowed to have different degrees of the LIV. He also pointed out that the modified relation of energy and momentum can affect mildly the detection of high energy radiation. Observations of $\gamma$-rays are based on pair creation in the detector material, on electromagnetic cascading in emulsion chambers for electrons (satellite experiments) or on the detection of Cherenkov light from air showers initiated by $\mathrm{TeV}$ photons in ground-based observations.

The conclusion of Ref. [9] is that LIV affects significantly the GZK and TeV- $\gamma$ thresholds but the effect of the modified dispersion relation on atmospheric interactions of the relevant high-energy particles is negligible.

The aim of the present paper is to discuss the possibility that deformed dispersion relations due to LIV could change not only the thresholds of some reactions at extremely high energies, but will also strongly affect the electromagnetic cascade development in the atmosphere and detectors. The reason for this is that deformed dispersion relations affect the so-called formation length of bremsstrahlung and pair production and, hence, their cross sections.

\section{Formation length and LI violation}

The longitudinal momentum transfer between highly relativistic interacting particles (photons or electrons) and the target nucleus is small. According to the uncertainty principle the interaction takes place over a long distance (formation length, coherence length). It appears in classical and quantum calculations for most electromagnetic processes (pair production, bremsstrahlung, Cherenkov radiation, synchrotron radiation, transition radiation). 
For bremsstrahlung

$$
q_{\|}=p_{e}-p_{e}^{\prime}-k / c
$$

where $p_{e}$ and $p_{e}^{\prime}$ are the electron momenta before and after the interaction and $k$ is the photon energy. For $E \gg m_{e} c^{2}$ we can neglect emission angles and then $q_{\|} \sim \frac{m^{2} c^{3} k}{2 E(E-k)}$. For pair production $q_{\|} \sim \frac{m^{2} c^{3} k}{2 E(k-E)}$. The formation length for bremsstrahlung is:

$$
l_{0} \sim \frac{\hbar}{q_{\text {॥ }}}=\frac{2 \hbar E(E-k)}{m^{2} c^{3} k}
$$

For pair production $E-k$ in the numerator is replaced by $k-E$.

While the transverse momentum exchanged with the nucleus, $q_{\perp}$, is of order $m c, q_{\|}$is very small and $l_{0}$ has macroscopic dimensions. For example, for a 25 GeV electron, emitting a $100 \mathrm{MeV}$ photon, $q_{\|} \sim 0.03 \mathrm{eV} / \mathrm{c}$ and $l_{0} \simeq 10 \mu \mathrm{m}$. For $10^{9} \mathrm{GeV}$ electron and $10^{5} \mathrm{GeV}$ bremsstrahlung photon $q_{\|} \sim 1.45 \times 10^{-8}$ $\mathrm{eV} / \mathrm{c}$ and $l_{0} \sim 14 \mathrm{~m}$.

The formation length increases with energy. The amplitude of the radiation is proportional to $l_{0}$, and its intensity is proportional to $\sim l_{0}^{2}$. If there is emission from an electron traversing a distance $D$, the trajectory acts as $\frac{D}{l_{0}}$ independent emitters, giving a total radiation intensity proportional to $\left|l_{0}\right|^{2} D / l_{0} \sim l_{0}$. [12]

The factors that could reduce $l_{0}$, and hence the probability for radiation or pair production are (see[12] and references therein) multiple scattering (LPM effect), photon interaction with the medium (dielectric suppression), external magnetic field (magnetic suppression), suppression of bremsstrahlung by pair production, and vice-versa.

It is interesting to note that LIV dispersion relation acts like the suppression factors mentioned above. For example, multiple scattering on the formation length (LPM effect) leads to an additional term in the expression for $q_{\|}$which increases the longitudinal momentum transfer. In the small angle approximation

$$
q_{\|} \simeq \frac{m^{2} c^{3} k}{2 E(E-k)}+\frac{k \theta_{M S}^{2}}{2 c}
$$

where $\theta_{M S}$ is the electron multiple scattering angle in half the formation length [12]. The increase of $q_{\|}$reduces the formation length.

Let us put, similarly to Eq. 1, the square of the modified momentum 


$$
q^{2}=p^{2}+\xi \frac{E^{3}}{E_{0} c^{2}}
$$

Then the Eq. 2 becomes $\left(q \simeq p+\xi \frac{E^{2}}{2 E_{0} c}\right)$

$$
q_{\|} \simeq \frac{m^{2} c^{3} k}{2 E(E-k)}+\xi_{e} \frac{E^{2}}{2 E_{0} c}-\xi_{e} \frac{(E-k)^{2}}{2 E_{0} c}-\xi_{\gamma} \frac{k^{2}}{2 E_{0} c}
$$

for bremsstrahlung, and

$$
q_{\|} \simeq \frac{m^{2} c^{3} k}{2 E(k-E)}+\xi_{\gamma} \frac{k^{2}}{2 E_{0} c}
$$

for pair production. In Eq. 7 LIV dispersion relation is used only for the particle with the highest energy - the photon.

The effect depends on the sign of the parameters $\xi$. If we take the electron and positron energies in Eq. 7 equal to $\frac{k}{2}(k$ is the photon energy) then

$$
q_{\|}^{\min } \simeq \frac{2 m^{2} c^{3}}{k}+\xi_{\gamma} \frac{k^{2}}{2 E_{0} c}
$$

When $\xi_{\gamma}<0 \quad q_{\|}^{\text {min }}$ becomes negative at the critical energy $k_{c r}=\left[\frac{4\left(m c^{2}\right)^{2} E_{0}}{\xi_{\gamma}}\right]^{\frac{1}{3}} \simeq$ $2.2 \times 10^{13} \mathrm{eV}$ for $\left|\xi_{\gamma}\right|=1$.

Let us now calculate the so-called suppression factor $S$, defined as:

$$
S=\frac{\frac{d \sigma}{d E}}{\frac{d \sigma}{d E_{B H}}}=\frac{l_{f}}{l_{0}}
$$

where $d \sigma / d E$ is the pair production differential cross section, $d \sigma / d E_{B H}$ is the Bethe-Heitler $(\mathrm{BH})$ cross section, $l_{f}$ is the formation length with suppression. The definition of $S$ is convenient for estimation of suppression due to different factors because it is easy to estimate the change of the quantity $q_{\| 1}$ caused by these factors and then to calculate $l_{f}$. For example, when suppression is due to multiple scattering and the suppression effect is strong, $S_{L P M}=\sqrt{\frac{E_{L P M} k}{E(k-E)}}$ (the material dependent energy $E_{L P M}$ is defined in[13]). For $E \approx k-E \approx k / 2$ $S_{L P M} \simeq 2 \sqrt{\frac{E_{L P M}}{k}}$.

In case of LIV

$$
S_{L I V}=\frac{1}{1+\xi_{\gamma} \frac{k^{3} u(1-u)}{E_{0}\left(m c^{2}\right)^{2}}}
$$

(here $u=E / k$ ) and for $E \approx k-E \approx k / 2$ 
Table 1

Suppression factors for pair production

\begin{tabular}{|l|lll|}
\hline$k(\mathrm{TeV})$ & $S_{L I V}$ & $S_{L P M}^{\text {air }}$ & $S_{L P M}^{P b}$ \\
\hline 1 & 1 & 1 & 1 \\
10 & 0.91 & 1 & 1 \\
100 & $10^{-2}$ & 1 & 0.41 \\
1000 & $10^{-5}$ & 1 & 0.13 \\
\hline
\end{tabular}

Table 2

Enhancement factor for pair production

\begin{tabular}{|l|l|}
\hline$k(\mathrm{TeV})$ & $S_{L I V}$ \\
\hline 0.01 & 1 \\
0.1 & 1 \\
1 & 1 \\
10 & 1.11 \\
15 & 1.2 \\
20 & 4.27 \\
\hline
\end{tabular}

$$
S_{L I V} \simeq \frac{1}{1+\xi_{\gamma} \frac{k^{3}}{4 E_{0}\left(m c^{2}\right)^{2}}}
$$

Neglecting 1 in the denominator for strong suppression, Eq.10 becomes

$$
S_{L I V} \simeq \frac{4 E_{0}\left(m c^{2}\right)^{2}}{\xi_{\gamma} k^{3}}
$$

Some numerical values for $S_{L I V}$ for pair production $\left(E_{0}=10^{28} \mathrm{eV}, \xi_{\gamma}=\right.$ 1 ) are shown in table 1. For comparison LPM suppression factors for air $\left(E_{L P M}=2.34 \times 10^{8} \mathrm{GeV}\right.$ at sea level $)$ and lead $\left(E_{L P M}=4.3 \times 10^{3} \mathrm{GeV}\right)$ are also shown in the table (the values for $E_{L P M}$ are taken from[12]).

If $\xi_{\gamma}=-1$

$$
S_{L I V}=\frac{1}{1-\frac{k^{3}}{4 E_{0}\left(m c^{2}\right)^{2}}}
$$

$S_{L I V}$ turns into enhancement, which becomes infinite for $k=k_{t h r} \equiv\left[4 E_{0}\left(m c^{2}\right)^{2}\right]^{\frac{1}{3}} \approx$ $2.2 \times 10^{13} \mathrm{eV} \equiv 22 \mathrm{TeV}$, as shown in table 2 .

This means that when $\xi$ is negative the formation length of the process (pair 
Table 3

Suppression factors for bremsstrahlung

\begin{tabular}{|l|lll|}
\hline$E(T e V)$ & $S_{L I V}$ & $S_{L P M}^{a i r}$ & $S_{L P M}^{P b}$ \\
\hline 1 & 1 & 1 & $6.6 \times 10^{-2}$ \\
10 & 0.57 & 1 & $2.1 \times 10^{-2}$ \\
100 & $1.3 \times 10^{-3}$ & 1 & $6.6 \times 10^{-3}$ \\
1000 & $1.3 \times 10^{-6}$ & 0.48 & $2.1 \times 10^{-3}$ \\
\hline
\end{tabular}

production in this case), respectively the cross section, increases. This sharp increase around $k_{t h r}$ could be compensated by multiple scattering and other suppression factors.

Results for bremsstrahlung are similar to those for pair production. If we neglect the last term in (6) (we suppose that LIV is negligible for low energy bremsstrahlung photons) the suppression factor becomes

$$
S_{L I V}=\frac{1}{1+\xi_{e} \frac{2 E^{3}(1-u)}{E_{0}\left(m c^{2}\right)^{2}}}
$$

or, for strong suppression,

$$
S_{L I V} \simeq \frac{E_{0}\left(m c^{2}\right)^{2}}{2 \xi_{e} E^{3}(1-u)}
$$

The corresponding LPM suppression factor (also for strong suppression) is

$$
S_{L P M}=\sqrt{\frac{E_{L P M}}{E} \frac{u}{1-u}}
$$

Some numerical values for $\xi=1$ and $u=0.001$ are compared in table 3 .

\section{Discussion and conclusions}

In general, dispersion relation with LIV which was proposed to explain the detection UHECR and $20 \mathrm{TeV} \gamma$-rays leads also to a reduction or to an increase of the formation length, depending on the sign of $\xi_{\gamma}$. This affects strongly the bremsstrahlung and pair production cross sections. A detailed analysis of the Plank-scale-motivated LIV scheme we used in this work, is made in [9]. Using the UHECR and the TeV- $\gamma$ data, as well as the upper bounds on time-of-flight differences between photons of different energies, the authors obtain the constraints on the parameter space. More general class of dispersion

relations has been concidered which in the high-energy regime takes the form 
$E^{2}-p^{2}-m^{2} \simeq \eta E^{2}\left(\frac{E}{E_{0}}\right)^{\alpha} \simeq \eta p^{2}\left(\frac{E}{E_{0}}\right)^{\alpha}$ where $\alpha$ and $\eta$ are free parameters $(c=1)$ of LIV.

In our work we analyzed the case $\alpha=1$ and $|\eta|=1$. According to[9] positive values of $\eta$ should be excluded which means that in our case $\xi$ must be positive. The critical photon energy $k_{c r}$ is of the same order of magnitude as the critical energy $E_{c}$ defined in [10] (see also [14]). In the case of LIV $E_{c}$ is obtained from the condition for a minimum target photon energy for pair creation in a soft photon field. Above $E_{c}$ the target photon energy becomes negative (for $\xi<0$ ) or grows (if $\xi>0$ ) until the Universe becomes transparent to ultrahigh energy photons . This reflects the fact that $([11,15])$ one can expect deviations from standard kinematics when the last two terms in the dispersion relation $E^{2} \approx p^{2}+m^{2}+\xi p^{3} / E_{0}$ are of comparable magnitude. For $\xi=1$ the condition becomes $p_{\text {dev }} \sim\left(m^{2} E_{0}\right)^{\frac{1}{3}} \sim 10 \mathrm{TeV}$.

The values obtained for the suppression factor $S$ and positive $\xi$ show that deformed LIV kinematics due to LIV dispersion relations strongly suppresses pair production and bremsstrahlung above some critical energy. The suppression is much stronger than in LPM. For the values of $\alpha$ and $\eta$ used above, the critical energy is relatively low and, unlike the LPM effect, the LIV suppression does not depend on the medium. The suppression factor decreases very rapidly with increasing energy, proportionally to $E^{-\frac{1}{3}}$. This will make photons and electrons very penetrating particles and will drastically suppress the electromagnetic shower development. For example, only about $20 \%$ of 100 $\mathrm{TeV}$ primary photons will interact in the atmosphere. For $\gtrsim 300 \div 400 \mathrm{TeV}$ photons the atmosphere will be transparent. Such an effect must have already been observed in the numerous experiments in cosmic rays. The depth of maximum in electromagnetic showers $X_{\max }$ is proportional to the product of the radiation length $X_{0}$ and the logarithm of the primary energy $\ln E$. In the case of LIV the radiation length $X_{0}$ above the critical energy becomes infinite, which changes drastically the behavior of electromagnetic and of hadronic air showers. This is especially true for giant air showers where the depth of maximum $X_{\max }$ is generally determined by the electromagnetic cascades of primary energy exceeding $10^{17} \mathrm{eV}$. Thus LIV with the parameters discussed above would contradict to the results from giant air showers [16].

Coleman and Glashow [1] have suggested a different scheme for LIV in which the maximum attainable velocity $c_{a}$ of a particle is different from the photon velocity $c$. The relevant dispersion relations then has the form $E_{a}^{2}=p^{2} c_{a}^{2}+$ $m_{a}^{2} c_{a}^{4}$. In this case our results will be applicable by substituting $c^{2}-c_{a}^{2}$ for $\xi \frac{E}{E_{0}}$ in (1). Then the critical energy will be defined as $k_{c r}=\frac{\sqrt{8} m_{e}}{\left|c^{2}-c_{e}^{2}\right|^{1 / 2}}$. If the shower development is observed with no deviation from the standard cascade theory up to $\simeq 22 \mathrm{TeV}$, this will put the limits $E_{0} \gtrsim 10^{28} \mathrm{eV}$ (Planck mass) or $\left|c^{2}-c_{e}^{2}\right| \lesssim 1.5 \times 10^{-15}$. 
The observations of giant atmospheric showers created by particles with energies $\gtrsim 10^{20} \mathrm{eV}$ give, in principle, the possibility for a more precise test of LIV. For example, if the case $\alpha=1$ must be ruled out, one can move to the $\alpha=2$ case, i.e. quadratic suppression of $E_{0}$. The suppression factor for pair creation then becomes $S_{L I V} \simeq \frac{4 E_{0}^{2}\left(m c^{2}\right)^{2}}{\xi k^{4}}$ and the drastic deformation of theshower development would be observed at energies $\gtrsim 10^{17} \mathrm{eV}$. In the frame of Coleman and Glashow scheme this will put the limits $\left|c^{2}-c_{e}^{2}\right| \lesssim 10^{-23}$. In this connection we would like to point out the interesting work[17] where new constraints on $\left|c_{\gamma}-c_{\pi^{0}}\right|\left(c_{\pi^{0}}\right.$ is the maximal attainable speed of neutral pions) are obtained by comparing the experimentally measured position of the shower maximum $X_{\max }$ with calculations.

Acknowledgements. The work of TS is supported in part by the US Department of energy contract DE-FG02 91ER 40626.

\section{References}

[1] S. Coleman and S. Glashow, Phys. Rev. D59 (1999) 116008.

[2] K. Greisen, Phys. Rev. Lett. 16 (1966) 748; G.T. Zatsepin and V.A. Kuzmin, Pis'ma JETP 4 (1966) 114.

[3] R.J. Protheroe and N. Meyer, Phys. Lett. B493 (2000) 1.

[4] F. Aharonian, astro-ph/0112314.

[5] V. Berezinsky, astro-ph/0107306.

[6] D.A. Kirzhnitz and V.A. Chechin, Pis'ma JETP, 14 (1971) 261.

[7] H. Sato and T. Tati, Prog. Theor. Phys. 47 (1972) 1788.

[8] L. Gonzalez-Mestres, physics/9704017; Proc.25th ICRC, Durban, 6 (1997) 113.

[9] G. Amelino-Camelia and T. Piran, Phys. Rev. D64 (2001) 036005; G. AmelinoCamelia, J. Ellis, N.E. Mavromatos, D.V. Nanopoulos, and S. Sarkar, Nature, 393 (1998) 763.

[10] T. Kifune. Ap.J. 518 (1999) L21.

[11] R. Aloisio, P. Plasi, P. Ghia, and A. Grillo, Phys. Rev. D62 (2000) 053010.

[12] S. Klein. Rev.Mod.Phys. 71 (1999) 1501.

[13] T. Stanev, H. Vankov, R.E. Streitmatter, R.W. Ellsworth and T. Bowen, Phys.Rev. D25 (1982) 1291.

[14] G. Sigl, Lect. Notes Phys. 556 (2000) 259. 
[15] S. Liberati, T.A. Jacobson and D. Mattingly, hep-ph/0110094; T. Jakobson, S. Liberati, and D. Mattingly, hep-ph/0112207.

[16] M. Nagano and A.A. Watson, Rev. Mod. Phys. 72 (2000) 689.

[17] E.E. Antonov, L.G. Dedenko, A.A. Kirillov, T.M. Roganova, G.F. Fedorova, and E.Yu. Fedunin, Pis'ma JETP 73 (2001) 506. 